\title{
Systematic Accounts on Percoid Fishes of Manimala River of Travancore
}

\author{
Mathews Plamoottil* \\ Asst. Professor \& HOD, Dept. of Zoology, Baby John Memorial Govt. College, Chavara, Kollam \\ Kerala
}

\begin{abstract}
The present study was conducted to analyze the taxonomic aspects of percoid fishes of Manimala River of Travancore, Kerala, India. Systematic studies were conducted on the fishes of the genera Ambassis, Parambassis, Etroplus, Pseudetroplus, Pristolepis, Anabas, Channa, Glossogobius, Awaous and Pseudosphromenus. The fishes were examined for meristic counts and metric characters and various taxonomic aspects were discussed..
\end{abstract}

Keywords: Taxonomy, Percomorphs, Travancore, Meristic counts, Metric characters

\section{INTRODUCTION}

Perciformes, the largest order of vertebrates (Nelson, 2006), containing about $41 \%$ of all bony fishes. It contains about one third of all fish species (Talwar and A. Jhingran, 1991). They belong to the class of ray-finned fishes and comprise over 10,000 species found in almost all aquatic ecosystems. They are food fishes or game fishes or aquarium fishes. Perciformes means "perch-like". Fishes of the order Perciformes or Percomorphi or Acanthopteri are known as Percoid fishes or Percoidean fishes or Perciform fishes or Perches.

In percoid fishes dorsal and anal fins are divided into anterior spiny and posterior soft-rayed portions, which may be partially or completely separated. Spinous dorsal fin well developed; soft dorsal similar to that of soft anal; the pelvic fins usually have one spine and up to five soft rays, positioned unusually far forward under the chin or under the belly; scales are usually ctenoid.

Even though a few surveys (Lakshmi, 2009; Plamoottil, 2015a) and taxonomic studies (Plamoottil, 2015b, 2016) conducted on the fish fauna of Manimala River, no serious attention was given to the systematics of the percoid fishes in it. It is an endeavor to study some taxonomic aspects of Perciform fishes of middle level regions of Manimala River.

\section{MATERials AND Methods}

Study Site: The Study was conducted during the period from January 2010 to December 2013. Chenapady, Manimala, Kottangl, Kulathurmoozhy, Thelapuzha, Kavanalkadavu, Keezhvaipur, Komalom and Karuthavadasserikara were the mid level sites of the Manimala River selected for the present study (Fig. 1- 9). Middle level regions of Manimala River basin consist of agricultural areas and deciduous forests. Riparian vegetation is moderately dense to thick. River bed is generally rocky in upper parts of middle level regions; but sand deposits can be seen within pools in this stretch. The bed materials are generally sandy gravel, gravelly sand and gravel in lower parts of middle level regions of the river. Pebbles and cobbles are present in sediments in appreciable amounts; but gravelly sediments are predominant. Some lower areas host a thick deposit of sand and gravel.

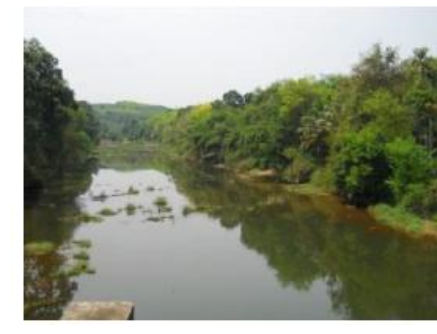

Fig. 1. Chenappady

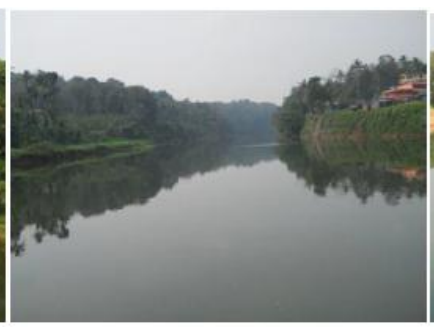

Fig. 2. Manimala

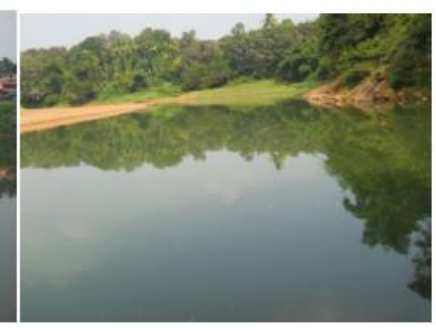

Fig. 3. Kottangal 


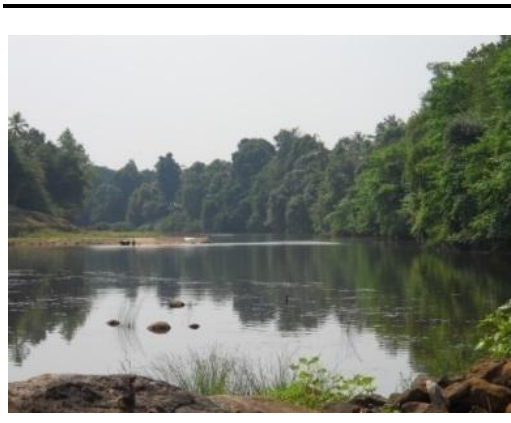

Fig. 4. Kulathoormuzhy

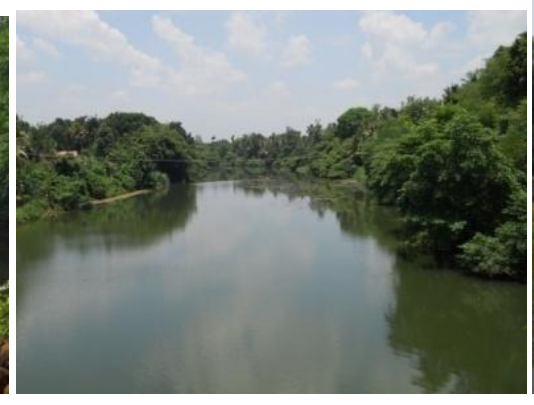

Fig. 5. Thelapuzha

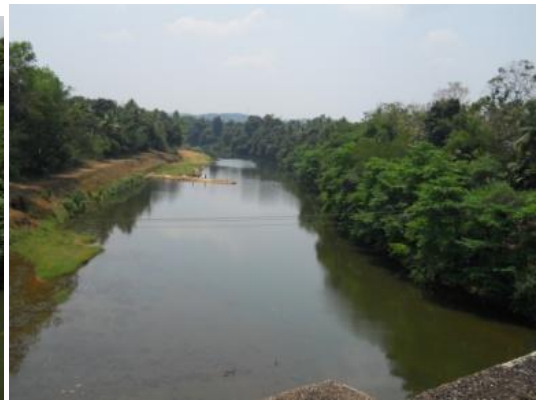

Fig. 6. Kavanalkadavu

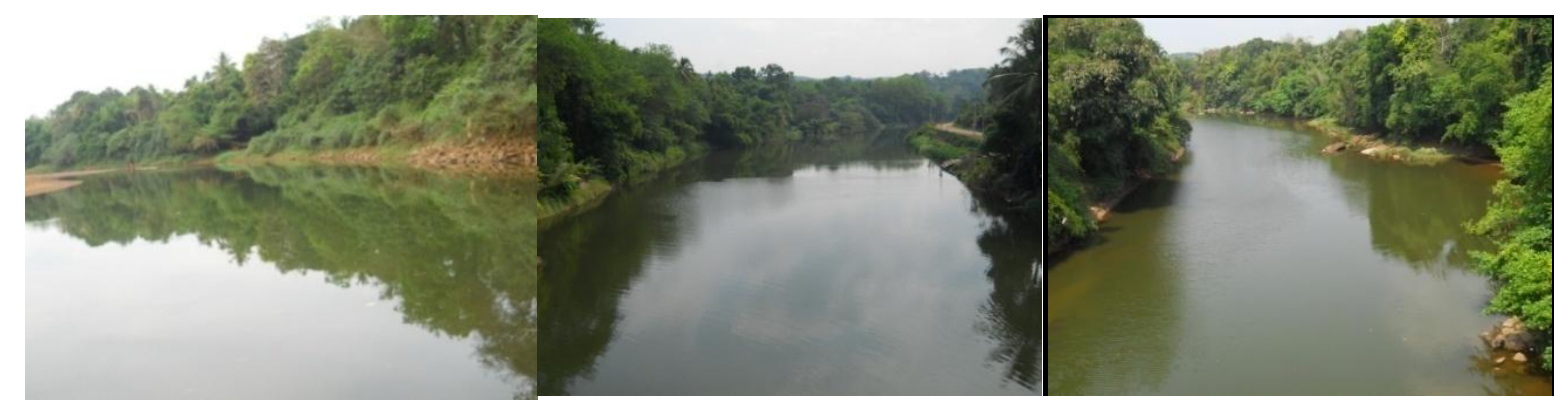

Fig. 7. Keezhvaipur

Fig. 8. Komalam

Fig. 9. Karuthavadasserikkara

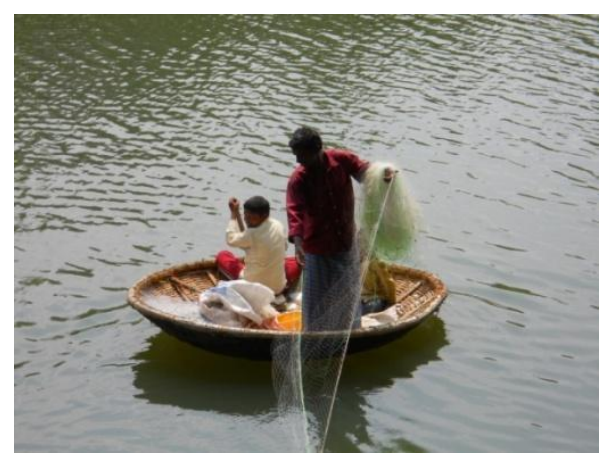

Fig. 10. Gill Fishing from a Kotta thoni

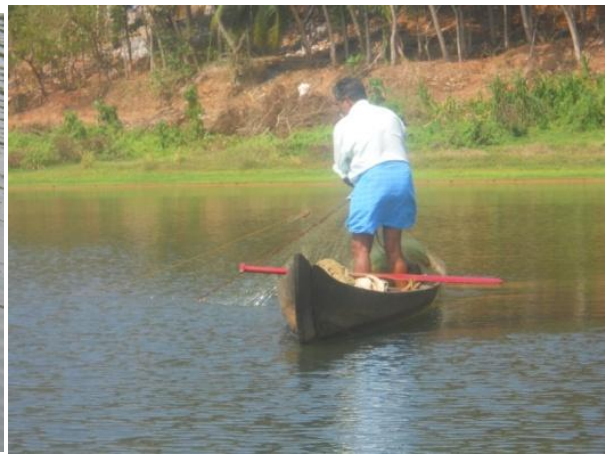

Fig. 11. Gill Fishing from a Vallom

Fish Collection and Preservation: Different types of nets are employed for fish collection. Dip nets, Gill nets (Fig. 10 \& Fig. 11) and Caste nets were used for this purpose. Nine percent formalin was used as preservative.

Live fishes were killed and fixed in formalin as they die in solution with all the fins expanded. Smaller specimens (less than $10 \mathrm{~cm}$ long) were directly put in formalin solution, while medium sized (10- $30 \mathrm{~cm}$ long), prior to the fixation be given a longitudinal incision along the abdomen, without injuring the alimentary canal. Large forms (fishes longer than $30 \mathrm{cms}$ ), be injected ten percent formalin in to the muscle and the abdomen, where the abdomen is not rounded but with a keel, the incision was made on the left side of the fish.

Identification: Methods used are of Jayaram (2002 \& 2010) and measurements followed standard practices. Taxonomic identification is based mainly on meristic, morphometric and descriptive characters. Meristic counts or countable characters of body such as fin ray counts and scale counts are precisely counted. Morphometric characters are those which can be measured. The proportion of the measurement of one part of the body in relation to other parts is worked out. The measurement of a part of body is worked out as percentage of the measurement of the other parts of the body or of percentage of head length or standard length of the body.

Head length, standard length and total length are mentioned in mm length and measurement of other parts of body is counted as measurements in percentage of head length and standard length. For confirming the identification and proving the identity of fishes collected, detailed description of a species is necessary. Meristic characters such as fin ray counts, position of eyes, fins, shape of mouth, lips etc were all taken in detail. Various body measurements were taken from many similar specimens. Numbers of characters used, set of measurements and counts were different for different group of fishes. 
Abbreviations Used: A- Anal fin rays; BDD- Body depth at dorsal fin origin; BDA- Body depth at anal fin rigin; BWD-Body width at dorsal; BWA- Body width at anal fin; BWSOA- Body width at soft anal fin; C- Caudal fin rays; CPS- Caudal peduncle scales; CR- Critically endangered; D- Dorsal fin rays; DD- Data deficient; DP-PL- Distance from pectoral fin to pelvic fin; DPL- A- Distance from pelvic to anal; DA-C- Distance from anal to caudal; DCP- Depth of caudal peduncle; DAV- Distance from anal to vent; DVV- Distance from ventral to vent; DOST- distance from occiput to snout; DODDistance from occiput to dorsal front; ED- Eye diameter; FF- Food fish; HL- Head length; HD- Head depth; HW- Head width; IOW- Inter orbital width; INW- Inter narial width; LP- Length of pectoral fin; LPL- Length of pelvic fin; LA- Length of anal fin; LC- length of caudal fin; LBD- Length of base of dorsal fin; LBA- Length of base of anal fin; LBC- Length of base of caudal fin; LC- Length of caudal peduncle; LSPD- Length of spinous dorsal fin; LSOD- Length of soft dorsal fin; LSOALength of soft anal fin; LBSPD- Length of base of spinous dorsal fin. LBSOD- Length of base of soft dorsal fin; LLS- Lateral line scales; LL/D- Scales between lateral line and dorsal fin; LL/V- Scales between lateral line and ventral fin; LL/A- Scales between lateral line and anal fin; L/Tr- Transverse scales; LRlc- Low risk least concerned; LRnt- Lower risk nearly threatened; OF- Ornamental fish; PPectoral fin rays; PRD- Predorsal length; POD- Post dorsal length; PRPL- Pre Pelvic length; PRAPre anal length; PRP- Pre pectoral length; PRPLS- Pre pelvic scales; PRAS- Pre anal scales; PDSPre dorsal scales; SL- Standard length; STL- Snout length; SLS- Scales in lateral series; VUVulnerable; WGM- Width of gape of mouth; WCP- Width of caudal peduncle.

\section{RESUlTS AND DisCUSSION}

Diversity of Percoid Fishes: Diversity is a measure of the degree of organization and efficiency with which energy, materials, space and time are used within a community (Payne, 1986). The degree of complexity of a community depends upon the number of species and the evenness with which individuals are distributed among species. The maintenance of diversity is of utmost importance in an ecosystem. Human interactions often result in the reduction of diversity within ecosystem, particularly through the loss of rare species.

During this study 15 percoid fishes were collected from various middle level locations of Manimala River. Ambassis commersoni, Parambassis dayi, Parambassis thomassi, Etroplus suratensis, Pseudetroplus maculatus, Pristolepis malabaricus, P. rubripinnis, Anabas testeudinus, Channa striatus, Channa marulius, Channa gachua, Channa micropeltes, Glossogobius giuris, Awous gutum and Pseudosphromenus cupanus are the percoid fishes collected from Manimala River. Most of them have different synonyms, common names and local names. Details of percoid fishes of Manimala River are shown in Table $1 \& 2$ and Fig. 12.

Most of the percoid fishes of the Manimala River are edible and popular food fishes. Some of them are also used as ornamental fishes. Etroplus suratensis and Channa marulius are commercially important and most palatable food fishes of the River. Glossogobius giurius are eaten only by some of the local inhabitants. Channa gachua and Pseudosphromenus cupanus are inedible.

Table1. Author, locality and classification of Percoid fishes of Manimala River

\begin{tabular}{|l|l|l|l|l|}
\hline Sl. No & Name of fish & Author & Type locality & Family \\
\hline 1 & Ambassis commersoni & Cuvier \& Valenciennes & Pondicherry & Ambassidae \\
\hline 2 & Parambassis thomassi & Day & Calicut \& Mangalore & Ambassidae \\
\hline 3 & Parambassis dayi & Bleeker & Malabar & Ambassidae \\
\hline 4 & Etroplus suratensis & Bloch & Surat & Cichlidae \\
\hline 5 & Pseudetroplus maculatus & Bloch & India & Cichlidae \\
\hline 6 & Glossogobius giuris & Hamilton- Buchanan & Gangetic provinces & Gobiidae \\
\hline 7 & Awauos gutum & Hamilton- Buchanan & Padma River & Gobiidae \\
\hline 8 & Pseudosphromenus cupanus & Valenciennes & Pondicherry & Belontidae \\
\hline 9 & Pristolepis malabartcus & Gunther & Mundakkayam & Pristolepididae \\
\hline 10 & Pristolepis rubripinnis & Britz et al. & Edathua & Pristolepididae \\
\hline 11 & Anabas testeudineus & Bloch & Java & Anabantidae \\
\hline 12 & Channa striatus & Bloch & Malabar & Channidae \\
\hline 13 & Channa marulius & Hamilton- Buchanan & Gangetic provinces & Channidae \\
\hline 14 & Channa gachua & Bloch \& Schneider & Bengal & Channidae \\
\hline 15 & Channa micropeltes & Cuvier & Java & Channidae \\
\hline
\end{tabular}




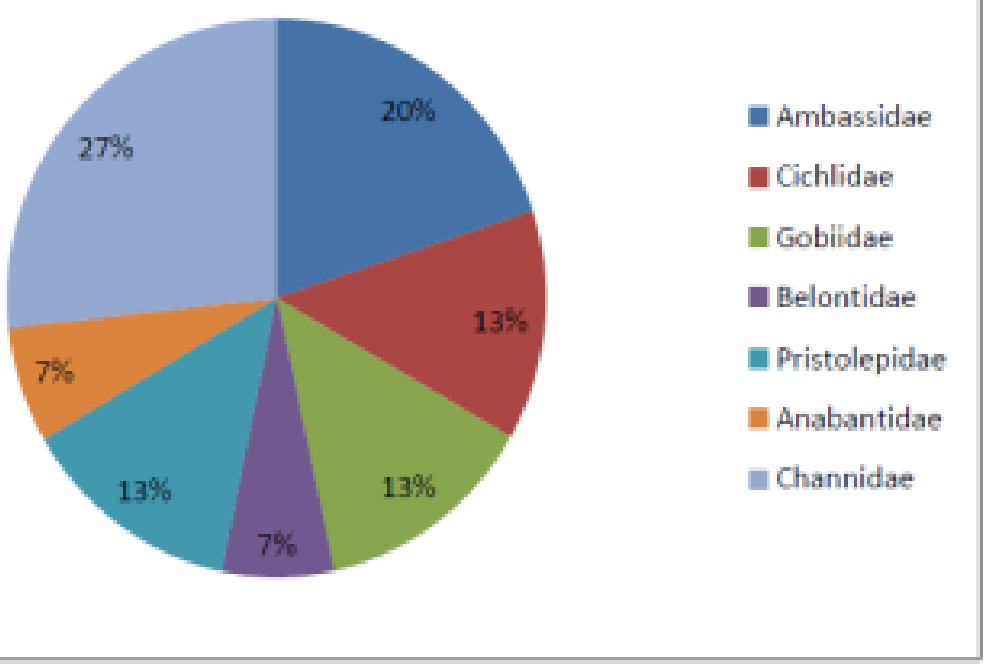

Fig12. Pie Diagram Showing the Percentage of Different Families of Perciform Fishes in Mid Level Regions of Manimala River

Table2. Names, significance and status of Percoid fishes of Manimala River

\begin{tabular}{|c|c|c|c|c|c|c|}
\hline $\begin{array}{l}\text { Sl. } \\
\text { No }\end{array}$ & Name of fish & Synonyms & $\begin{array}{l}\text { Common } \\
\text { name/Trade } \\
\text { name }\end{array}$ & $\begin{array}{l}\text { Local name } \\
\text { (Malayalam) }\end{array}$ & Significance & Status \\
\hline 1 & $\begin{array}{l}\text { Ambassis } \\
\text { commersoni }\end{array}$ & Chanda ambassis & $\begin{array}{l}\text { Commerson's } \\
\text { glassy } \\
\text { perchlet }\end{array}$ & $\begin{array}{l}\text { Nandan, } \\
\text { Mulli, } \\
\text { Arinjil, } \\
\text { Aranjeen, } \\
\text { Veloori }\end{array}$ & $\mathrm{FF}, \mathrm{OF}$ & DD \\
\hline 2 & Parambassis dayi & $\begin{array}{l}\text { Chanda ranga } \\
\text { Ambassis ranga, }\end{array}$ & $\begin{array}{l}\text { Indian glassy } \\
\text { fish, } \\
\text { High fin glass } \\
\text { fish }\end{array}$ & $\begin{array}{l}\text { Arinjil, } \\
\text { Veloori }\end{array}$ & $\mathrm{FF}, \mathrm{OF}$ & VU \\
\hline 3 & $\begin{array}{l}\text { Parambassis } \\
\text { thomassi }\end{array}$ & Chanda thomassi & $\begin{array}{l}\text { Giant glass } \\
\text { fish }\end{array}$ & Mullucheru & $\mathrm{FF}$ & LRnt \\
\hline 4 & $\begin{array}{l}\text { Pseudetroplus } \\
\text { maculatus } \\
\text { (Bloch) }\end{array}$ & $\begin{array}{l}\text { Chaetodon } \\
\text { maculatus }\end{array}$ & $\begin{array}{l}\text { Spotted } \\
\text { Etroplus, } \\
\text { Orange } \\
\text { chromide }\end{array}$ & $\begin{array}{l}\text { Pallathi, } \\
\text { Pootta }\end{array}$ & FF, OF & LRlc \\
\hline 5 & $\begin{array}{l}\text { Etroplus suratensis } \\
\text { (Bloch) }\end{array}$ & $\begin{array}{l}\text { Chaetodon } \\
\text { suratensis }\end{array}$ & $\begin{array}{l}\text { Banded Pearl } \\
\text { spot, } \\
\text { Striped } \\
\text { chromide, } \\
\text { Green } \\
\text { chromide } \\
\end{array}$ & Karimeen & $\mathrm{FF}, \mathrm{OF}$ & LRlc \\
\hline 6 & $\begin{array}{l}\text { Pristolepis } \\
\text { malabaricus }\end{array}$ & $\begin{array}{l}\text { Pristolepis } \\
\text { malabarica, } \\
\text { Catopra } \\
\text { malabarica }\end{array}$ & $\begin{array}{l}\text { Malabar sun } \\
\text { fish }\end{array}$ & Chutichi & $\mathrm{FF}, \mathrm{OF}$ & DD \\
\hline 7 & $\begin{array}{l}\text { Pristolepis } \\
\text { rubripinnis }\end{array}$ & Nil & $\begin{array}{l}\text { Red finned } \\
\text { catopra }\end{array}$ & Chembally & $\mathrm{FF}, \mathrm{OF}$ & DD \\
\hline 8 & $\begin{array}{l}\text { Anabas } \\
\text { testeudineus }\end{array}$ & $\begin{array}{l}\text { Anthias } \\
\text { testudineus } \\
\text { Anabas scandens } \\
\text { A.t. riveri, } \\
\text { A.t. lacustri } \\
\text { A.t. ricei }\end{array}$ & $\begin{array}{l}\text { Climbing } \\
\text { perch }\end{array}$ & $\begin{array}{l}\text { Undikolli, } \\
\text { Chovane- } \\
\text { kolli, } \\
\text { Andi kalli, } \\
\text { Karippidi, } \\
\text { Kaithakkora, } \\
\text { Kallemutti, } \\
\text { Kallada, } \\
\text { Karooppu }\end{array}$ & $\mathrm{FF}, \mathrm{OF}$ & VU \\
\hline
\end{tabular}


Systematic Accounts on Percoid Fishes of Manimala River of Travancore

\begin{tabular}{|c|c|c|c|c|c|c|}
\hline 9 & $\begin{array}{l}\text { Glossogobius } \\
\text { giuris (Hamilton- } \\
\text { Buchanan) }\end{array}$ & $\begin{array}{l}\text { Gobius giuris } \\
\text { Gobius gutum }\end{array}$ & $\begin{array}{l}\text { Goby, } \\
\text { Tank goby, } \\
\text { Bar-eyed } \\
\text { goby, } \\
\text { Sleeper goby }\end{array}$ & $\begin{array}{l}\text { Poolan, } \\
\text { Poosan, } \\
\text { Poan, } \\
\text { Poozhan } \\
\text { Kurudan, } \\
\text { Varatta- } \\
\text { poolan }\end{array}$ & $\mathrm{FF}, \mathrm{OF}$ & LRlc \\
\hline 10 & Awous gutum & $\begin{array}{l}\text { Gobius gutum, } \\
\text { Gobius striatus, } \\
\text { Awaous stamineus }\end{array}$ & $\begin{array}{ll}--- \\
\end{array}$ & Maaran & $\mathrm{OF}$ & LRlc \\
\hline 11 & $\begin{array}{l}\text { Pseudosphromenus } \\
\text { cupanus } \\
\text { (Valenciennes) }\end{array}$ & $\begin{array}{l}\text { Macropodus } \\
\text { cupanus } \\
\text { Polyacanthus } \\
\text { cupanus }\end{array}$ & $\begin{array}{l}\text { Spike tailed } \\
\text { paradise fish }\end{array}$ & $\begin{array}{l}\text { Karingana, } \\
\text { Wunntee }\end{array}$ & $\mathrm{FF}, \mathrm{OF}$ & LRlc \\
\hline 12 & $\begin{array}{lr}\text { Channa } & \text { gachua } \\
\text { (Bloch } & \& \\
\text { Schneider) } & \end{array}$ & $\begin{array}{l}\text { Ophiocephalus } \\
\text { gachua } \\
\text { Ophiocephalus } \\
\text { apus } \\
\text { Ophiocephalus } \\
\text { harcourt- butleri } \\
\text { Channa burmanica } \\
\text { Ophiocephalus } \\
\text { gachua kelaarti }\end{array}$ & $\begin{array}{l}\text { Asiatic snake } \\
\text { head }\end{array}$ & $\begin{array}{l}\text { Koravu, } \\
\text { Vattudi, } \\
\text { Vatton, } \\
\text { Vattakkannan, } \\
\text { Manathukan- } \\
\text { nan, } \\
\text { Cheran }\end{array}$ & $\mathrm{OF}$ & VU \\
\hline 13 & $\begin{array}{l}\text { Channa marulius } \\
\text { (Hamilton- } \\
\text { Buchanan) }\end{array}$ & $\begin{array}{l}\text { Ophiocephalus } \\
\text { marulius } \\
\text { Ophiocephalus } \\
\text { leucopunctatus } \\
\text { Ophiocephalus } \\
\text { pseudomarulius } \\
\text { Channa marulius } \\
\text { ara }\end{array}$ & $\begin{array}{l}\text { Giant snake } \\
\text { head } \\
\text { Peacock } \\
\text { snake head }\end{array}$ & $\begin{array}{l}\text { Cheran, } \\
\text { Cherumeen } \\
\text { Cheruvaral } \\
\text { Korava }\end{array}$ & $\mathrm{FF}, \mathrm{OF}$ & LRnt \\
\hline 14 & $\begin{array}{l}\text { Channa } \\
\text { micropeltes } \\
\text { (Cuvier) }\end{array}$ & $\begin{array}{l}\text { Ophiocephalus } \\
\text { micropeltes }\end{array}$ & $\begin{array}{l}\text { Malabar } \\
\text { snake head }\end{array}$ & $\begin{array}{l}\text { Vaaka,Vaaka } \\
\text { varal }\end{array}$ & FF, OF & $\mathrm{CR}$ \\
\hline 15 & $\begin{array}{l}\text { Channa striatus } \\
\text { (Bloch) }\end{array}$ & $\begin{array}{l}\text { Ophiocephalus } \\
\text { striatus }\end{array}$ & $\begin{array}{l}\text { Striped snake } \\
\text { head, Striped } \\
\text { murrel } \\
\text { Banded snake } \\
\text { head }\end{array}$ & $\begin{array}{l}\text { Bral, Varal, } \\
\text { Sowrah, } \\
\text { Kaunan }\end{array}$ & $\mathrm{FF}, \mathrm{OF}$ & LRlc \\
\hline
\end{tabular}

Species Accounts: Conservation efforts require scientific documentation of the bio- resources along with details on their distribution and status (Easa \& Shaji, 2003). This requires inventory and systematic studies of the fishes. A detailed study on the different aspects of taxonomy of percoid fishes is helpful in the correct identification and to search for the presence of new species.

\section{Ambassis commersoni Cuvier \& Valenciennes}

Ambassis commersoni Cuvier, Hist. Nat. Poiss., 2: 176 (type locality: Pondicherry) (Fig. 13 \& 14).

Meristic counts: D- VII; I, 11; V- I, 5; P- 12; A- III, 8; C- 18; LLS- 30/31; LL/D- 5.0; LL/V- 71/2; LL/A- 81/2; PRPLS- 8; PRAS- 17; CPS- 7.0; PDS- 14.

Metric characters: TL (mm)- 65.0; SL (mm)- 48.0; HL (mm)- 21.0. \% SL: HL-43.8; HD-27.1; HW18.8; BDD-45.8; BDA-39.6; BWD-18.8; BWA-16.7; PRD-43.8; POD-64.6; PRPL-39.6; PRA-62.5; LP-29.2; LPL-25.0; LA-18.8; LC-35.4; DP-PL-29.2; DPL- A-29.2; DA-C-41.7; LBD-41.7; LBA18.8; LBC-14.6; LCP-4.2; DCP-14.6; DAV-6.3; DVV-16.7; PRP-39.6. \% HL: HD- 62.0; HW-42.8; ED-33.3; IOW-23.8; INW-19.0; WGM-23.8; STL- 28.6.

Other features: No distinct notch between 2 dorsal fins; both these are interconnected; lower part of inter operculum and outer opercle serrated; tip of pectoral and pelvic fin reach anal front; no distinct serration around orbit; tiny black spots between $2^{\text {nd }}, 3^{\text {rd }}$ and $4^{\text {th }}$ dorsal spines; mouth terminal and upturned.

\section{Parambassis dayi (Bleeker)}

Ambassis dayi Bleeker, Natuurk. Verh. Holland.Maatsch. Wetensch. Haarlem, 2 (2): 95 (type locality: Malabar) (Fig. 15- 17). 
Diagnosis: Tip of pectoral and pelvic fin reach anal front; space between $2^{\text {nd }}$ and $3^{\text {rd }}$ dorsal spines dusky.

Meristic counts: D- VII, 12; P- 12; V-I, 5; A- III, 10; C- 17; LLS- 37; LL/D- 7; LL/V- 12; PDS- 18.

Metric characters: TL (mm)- 65.0; SL (mm)- 48.0; HL (mm) - 18.0. \% SL: HL-37.5; HD-25.0; HW12.5; BDD-31.3; PRD-45.8; POD-68.8; PRP-35.4; PRPL-38.5; PRA- 62.5; LD-29.2; LA-18.8; LC31.3; DPL- A- 27.1; LBD-35.4; LBA-23.0; DAV-6.3; DVV- 16.7. \% HL: HD- 66.7; HW-33.3; ED33.3; STL-27.8; IOW-22.2; WGM-16.7.

Remarks: The present fish is without a spine on the second dorsal fin; it is a major difference from its congeners.

\section{Parambassis thomassi (Day)}

Ambassis thomassi Day, Proc. Zool. Soc. London: 369 (type locality: Calicut and Mangalore) (Fig. 18-20).

Diagnosis: Body stout, deep and compressed. Lateral line continuous with 43 scales. Lower jaw longer. Lower edge of pre orbital denticulated.

Meristic counts: D- VII; I, 11; P- 14; V- I, 5; A- III, 10; C- 17; LLS- 43+ 2; L/Tr- 51/2/111/2; LL/D- 51/2; LL/V- 111/2; PDS- 17; CPS- 7; LLA- 101/2; PPLS- 6; PRAS-22.

Metric Characters: TL (mm)- 151.0; SL (mm)- 118.0; HL (mm) - 44.0. \% SL: HL-37.3; HD-27.1; HW-13.6; BDD-28.8; BDA-26.3; BWD-12.7; BWA- 10.2; PRD-44.1; POD- 56.8; PRP-35.6; PRPL37.3; PRA-61.0; LD-21.2; LP-22.9; LPL-18.2; LA-14.4; LBD-37.3; LBA-18.6; LCP-18.6; DCP-11.0; WCP-4.2. \% HL: HD-72.7; HW- 36.4; ED-27.3; IOW-18.2; INW-18.2; STL-34.1.

Other Features: Vertical margin of pre opercle finely serrated; its lower double edge more coarsely so, especially at the angle. Second dorsal spine longest; second anal spine equals the third. A prominent black line present near to the caudal region.

\section{Channa marulius (Hamilton- Buchanan)}

Ophiocephalus marulius Hamilton- Buchanan, 1822, Fishes of Ganges: 65, 367 (type locality: Gangetic provinces) (Fig. 21-23).

Diagnosis: A black white edged ocellus present on upper part of basal portion of caudal fin. Large black blotches adorn the sides of the long body. Sixty six scales present on the lateral sides of the body. Sixteen scales present before the dorsal fin.

Meristic characters: D- 49; P- 18; V-7; A- 32; C- 14; LLS- 66; PDS- 16.

Metric characters: TL (mm)- 240.0; SL (mm)- 200.0; \% SL; HL-32.5; BDD- 16.5; PRD-34.0; POD68.0; LCP- 8.0.

\section{Channa micropeltes (Cuvier)}

Ophiocephalus micropeltes Cuvier, Hist. Nat. Poiss., 7: 427 (type locality: Java) (Fig. 24- 26).

Diagnosis: Dorsal fin with 42 and anal fin with 25 rays; lateral line scales 102; minute brownish spots present on body.

Meristic counts: D- 42; V- I, 6; P- 16; A- 25; C- 14; LLS- 102; PDS- 22; CPS- 13.

Metric Characters: TL (mm)- 235.0; SL (mm)-193.0; HL (mm) - 66.0. \% SL: HL-34.2; HD-16.6; BDD-16.6; PRD-27.5; POD-69.0; PRP-36.8; LD-7.3; LP-12.0; LPL-10.8; LBD-62.2; LBA-36.8; LCP-10.4; DCP-10.4. \% HL: HD-48.5; ED-12.1; STL-22.7.

Other features: Dorsal and dorso lateral sides are greenish dark; ventral and ventro lateral sides yellowish; dorsal and caudal fins with a light dark color; pectoral and pelvic fin yellowish; eyes are protruding. Young one morphologically different to adults; in young ones 2 black horizontal stripes run from the tip of head to caudal tip; of these upper one starts from the middle of the eye and reach middle part of caudal fin; the lower stripe starts from the lower part of upper jaw and ends in the middle of caudal fin. Adults devoid of any lateral bands. 


\section{Channa striatus (Bloch)}

Ophiocephalus striatus Bloch, 1793, Natures. Ausland. Fische, (7): 141 (type locality: Malabar) (Fig. 27- 29).

Diagnosis: Seventeen scales present before dorsal fin; lateral line scales 56. Dark obligue bands pass from ventral surface upto the lateral line.

Meristic characters: D- 44; P- 15; V- 6; A- 25; C- 13; LLS- 56; LL/D- 41/2; LL/V- 7 1/2; PDS-17; LL/A- 11; CPS- 10.

Metric characters: TL (mm)- 220.0; SL (mm)- 180.0; \% SL: HL- 34.4; HD-25.6; BDD- 25.0; PRD34.4; LCP- 7.2; DCP-10.6; \% HL: ED- 12.9; HD- 74.2..

\section{Channa gachua (Bloch \& Schneider)}

Ophiocephalus gachua Hamilton- Buchanan, 1822, Fishes of Ganges: 68, 367, (type locality: Bengal) (Fig. 30- 32).

Diagnosis: Fourty scales present in longitudinal series; twelve scales present before the dorsal fin; a black ocellus is present at the end of dorsal fin.

Meristic characters: D- 28; P- 13; V- 5; A- 19; C- 11; LLS- 40; PDS- 12; LL/D- 3 1/2; LL/V- 5 1/2; LL/A- $51 \frac{1}{2}$.

Metric characters: TL (mm) - 77; SL (mm)- 62.0; \% SL: HL- 32.2; HD- 19.3; BDD- 14.3; PRD32.5; \% HL: HD- 60.0; ED- 15.0.

\section{Pseudosphromenus (Macropodus) cupanus (Valenciennes)}

Polycanthus cupanus Valenciennes, Hist. Nat. Poiss., 7: 357 (type locality: Ariancoupan River, Pondicherry) (Fig. 33- 35).

Diagnosis: Dorsal and anal fins long and provided with spiny and rayed portions; the former originates just above anal origin and extends posteriorly a little in front of post end of anal. Tip of pectoral and pelvic reach behind the origin of anal; a small black spot present at the base of caudal; first ray of pelvic elongated.

Meristic characters: D- XIII, 6; P- 10; PL- I, 5; A- XVI; 12; C- 6; LLS- 30; PDS- 30.

Metric characters: TL (mm)- 36; SL (mm)- 27; \%SL: HL- 37.0; HD-25.9; BDD- 22.3; PRD- 30.6; DCP- 5.6; \% HL: HD- 70.0; ED- 30.0

\section{Pseudetroplus maculatus (Bloch)}

Chaetodon maculatus, Bloch, 1785, Syst. Ichth: 427 (type locality: India) (Fig. 36- 38).

Diagnosis: Body with 3- 4 black blotches on the dorso- posterior half of the body; anal, dorsal, ventral and caudal fins have dark staining on the border. Lateral line incomplete.

Meristic characters: D- xvii- xx, 8-10; P- I, 15-16; V- i, 5; A- xii- xv, 8- 9.

Metric characters: TL (mm)-84.0; SL (mm)-61.0; \% SL: HL-65.6; HD-39.3; BDD-49.2; PRD-50.8; LCP-10.7; DCP-14.8; \% HL: HD- 39.3; ED- 9.8.

\section{Etroplus suratensis (Bloch)}

Chaetodon suratensis Bloch, 1785, 1785, Syst. Ichth: 427 (type locality: Surat) (Fig. 39- 41).

Diagnosis: Five to six slanting black bands present on the body; each scale with a fluorescent pearly spot on it. Pectorals with a large black spot at the base; dorsal with 18 spines; anal with 12 spines.

Meristic characters: D- XVIII, 15; P- 14; V- I, 5; A- XII, 13; C- 16;.LLS- 43; PDS- 13; LL/D- 81/2; LL/V- 18; LL/A- 201/2;

Metric characters: TL (mm)- 160.0; SL (mm)- 131.0; \% SL: HL- 30.5; HD- 40.5; BDD- 50.4; PRD42.0; LCP- 7.0; DCP- 15.3; \% HL: HD- 132.5; ED- 22.5.

\section{Pristolepis malabarica ( Gunther)}

Catopra malabarica Guenther, 1864, Ann. Mag. nat. Hist., (3) 14: 375 (type locality: hill ranges of Trvancore, Kerala). Pristolepis malabarica, Plamoottil \& Abraham, 2013, J. Adv. Zool. 34 (1): 2835 (Fig. 42- 44). 
Diagnosis: Pre opercle with its vertical limb roughened or slightly serrated; at the angle of pre opercle 3 or 4 small spines present; angle of inter and sub opercles generally without serrations but fine serrations present in a few cases; opercle with two sharp flat bifid spines; lateral line scales - 19-23/ 8-11; scales from lateral line to ventral fin- $8 \frac{1}{2} 2-10 \frac{1}{2}$.

Meristic counts: D- XIV- XV; 11- 12; P- 12- 14; V- I, 5; A- III; 8- 9; C- 14; LLS - 19- 23/ 8- 11; LL/D- 31/2; LL/V- 81/2- 101/2; L/Tr- 31/2/101/2; PDS 10- 13; CPS- 16- 18;

Metric characters: SL (mm)- 63.0- 88.0; HL (mm) - 22.0-31.0. \% SL: BDD- 41.4- 45.5; BDA-42.346.6; BWD- 21.1- 23.1; BWA- 12.7- 15.4; HL- 33.6- 36.5; HD-31.0- 40.9; HW-18.3- 20.8; PRD37.1- 40.9; POD-63.6- 68.3; PRP- 32.3- 38.1; PRPL- 40.0-45.7; PRA-69.2-74.6; LSPD- 11.4-14.3; LSOD-20.0-25.0; LP- 23.6-28.4; LPL-20.6-23.3; LSOA- 16.7-22.7; LBSPD- 43.2- 48.3; LBSOD13.6-20.0; LCP- 7.9- 13.6; DCP- 16.9- 18.5; WCP- 3.2- 6.2; DAV- 6.4- 7.7; DVV- 22.8- 26.2. \% HL: HD- 90.9- 96.8; ED- 25.8- 26.7; IOW- 26.1- 33.3; INW- 17.4- 22.2; STL-28.3- 30.6; WGM27.6- 31.1 .

Other Features: Opercle with two sharp flat bifid spines. Outer rows of teeth in jaws enlarged; parasphenoid bears fine teeth with rounded crowns; smaller teeth present also on the root of the tongue on the epi and cerato- hyals. Superior and inferior pharyngeals bear villiform teeth.

\section{Pristolepis rubripinnis Britz et al.}

Pristolepis rubripinnis, Britz et al. 2012, Zootaxa 3345: 59-68 (Type locality: Pamba and Chalakudy Rivers) (Fig. 45- 47).

Diagnosis: Fins orange red; three spines in anal fin and 14- 15 spines in dorsal fin; eyed reddish.

Meristic counts: D- XIV-XV, 13-14; P- 13-14; P- I, 5; A- III, 9; C- 14; LLS- 28-29; LL/D- 41/2; LL/V- 101/2; L/Tr- 41/2/101/2; PDS- 19-20; C- 41/2.

Metric characters: SL (mm)- 87-110; \% SL: BDD-43.00-43.68; BDA-44.00-45.9; BWD-20.69-23.00; BWA-11.82-17.24; HL- 34.54-39.08; HD-33.18-37.89; HW-20.69-22.00; PRD- 39.00-42.53; POD65.45-70.00; PRP-35.5-37.36; PRPL-41.82-42.00; PRA-71.00-78.16; LSPD-11.49-11.82; LSOD21.84-23.64; LP-24.5-25.45; LPL-21.82-25.29; LSOA-22.22-29.09; LBSPD-46.81-49.00; LBSOD14.94-18.18; LBA- 21.84-23.00; LCP-9.19-13.64; DCP- 4.00-17.27; WCP-3.00-4.09; DAV-5.91-6.8; DVV-26.36-31.03. \% HL: HD-95.59-98.65; HW-52.94-63.16; ED-23.53-26.32; IOW-28.95-29.73; INW-16.18-21.05; STL-21.62-26.32; WGM-23.03-27.63.

\section{Anabas testudineus (Bloch)}

Anthias testudineus, Naturges. Ausland. Fische, (6); 121 (type locality: Java) (Fig. 48- 50).

Diagnosis: 21- 29 scales present along the lateral series; operculum serrated with spines; mouth large with small conical teeth; a black spot present at the base of pectoral fin.

Meristic counts: D- XVIII, 9; A- XI, 9; P- 16; V- I, 5

Metric characters: TL (mm)- 127.0; SL (mm)- 101.0; HL (mm)- 34.0. \% SL: HL-33.7; HD-25.7; HW-23.8; BDD-31.7; BDSOA-28.7; BWD-21.8; BWSOA-9.9; PRD-35.6; POD-67.3; PRP-34.7; PRPL-40.6; PRA-57.4; LSOD-14.8; LP- 20.8; LPL-17.8; LSOA-13.8; LC-25.7; LBD-58.4; LBP-6.9; LBPL-4.9; LBA-36.6; LBC-14.8; LCP-8.9; DCP-14.8; WCP-3.9; DP-PL-9.9; DPL- A-18.8. \% HL: HD- 76.5; HW- 70.6; ED-20.6; IOW-35.3; INW-20.6; WGM-29.4; DOST- 73.5; DOD-35.2.

Other features: Body oblong and compressed at the posterior region; it is greenish to dark grey on dorsal side and flanks and ventral side pale yellow; a well marked blotch is seen at the base of caudal peduncle; pectoral and anal fins pale yellow; dorsal and caudal fins dark grey.

\section{Glossogobius giuris giuris (Hamilton- Buchanan)}

Gobius giuris giuris Hamilton- Buchanan, 1822, Fishes of Ganges: 51 (type locality: Gengetic provinces) (Fig. 51- 53).

Diagnosis: Dorsal fin divided into two; pelvic fin located on the ventral side of the body; head greatly depressed; eyes located on the dorsal side of head; dorsal fin located near to snout than caudal base.

Meristic characters: D- vi; i, 10; P- 19; v- 10; A- iii, 7; C- 14; L/tr- 14; SLS-34; PDS- 19; CPS- 7.

Metric characters: TL (mm)- 362.0; SL (mm)- 212.0; \%SL: HL-32.5; HD- 18.4; BDD- 11.3; PRD22.4; LCP- 16.0; \%HL: ED- 14.5; HD- 56.5. 
Remarks: It is the sleepy gopi; known as 'poolon'/ 'poosan'/ 'poozhan'/ 'pooan' in malayalam.

\section{Awaous gutum (Hamilton- Buchanan)}

Gobius gutum Hamilton- Buchanan, 1822, Fishes of Ganges,: 50, 366 (type locality: Padma River).(Fig. 54 \& 55)

Diagnosis: Pelvic fins are united and elongate and not adhered to the body; dorsal, pectoral and caudal fins are mottled with minute black dots.

Meristic counts: D- vi, 11; P- I, 14; V- 8; A- 12; C 14; LLS- 51, PDS- 16;

Metric characters: TL (mm)- 134.0; SL (mm)- 110.0; HL (mm)-37.0. \% SL: HL-33.6; BDD-27.3; PRD-40.0; POD-61.8;.8PRP- 32.7; PRPL- 31.8; PRA-63.6; LD-14.5; LBA-22.7; LBC-10.9; LC-21.8; DPL- A-32.7; LCP-18.2. \% HL: HD-56.8; HW- 67.6; ED-16.2; STL-51.3; IOW-24.3; WGM-40.5.

Other features: Dark blotches present on flanks; two dorsal fins present; first dorsal inserted a little in front of the origin of pelvic, a little nearer to snout than caudal fin; tip of pelvic fin reach anal opening; tip of anal fin very nearer to root of caudal base; caudal fin obtuse.

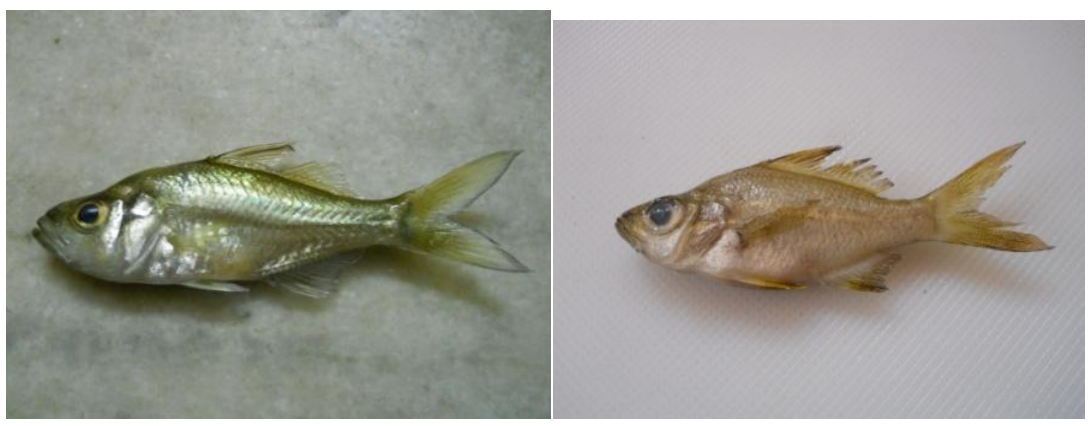

Fig. 13 Ambassis commersoni, greenish

Fig. 14 A. commersoni, Yellowish

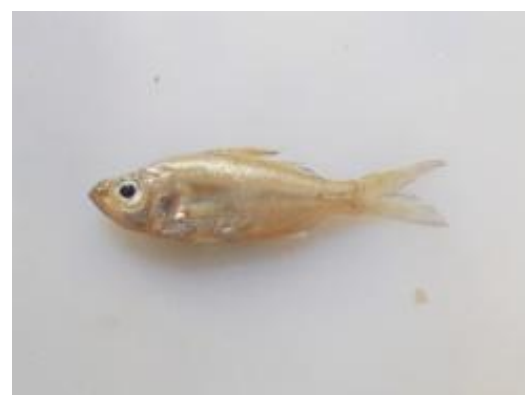

Fig.15. Parambassis dayi, silvery

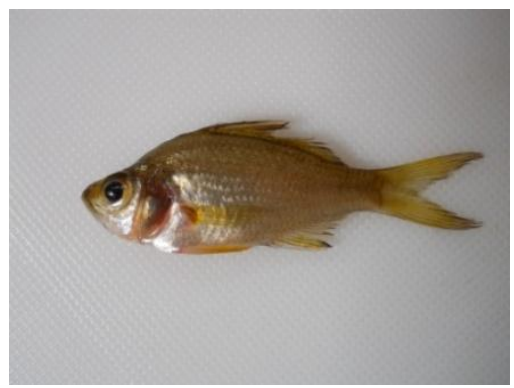

Fig.18. $P$. thomassi, golden;

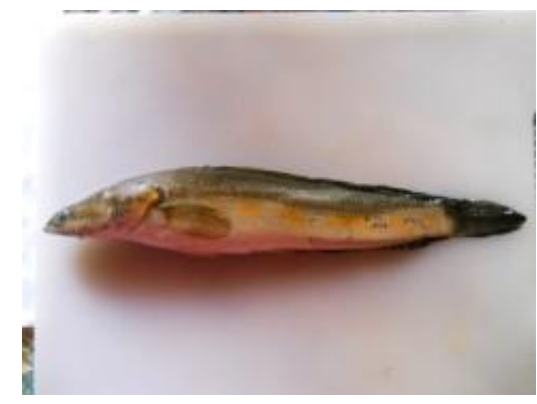

Fig.21. Channa marulius, yellowish;

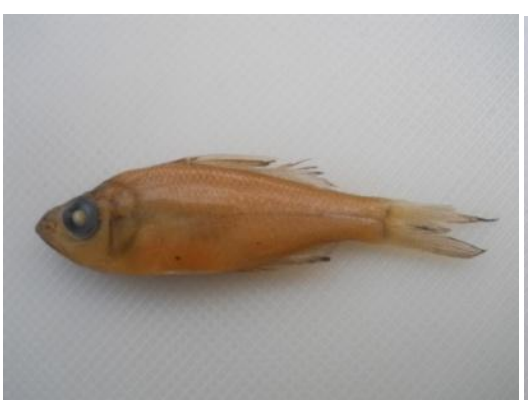

Fig.16. P. dayi, brownish

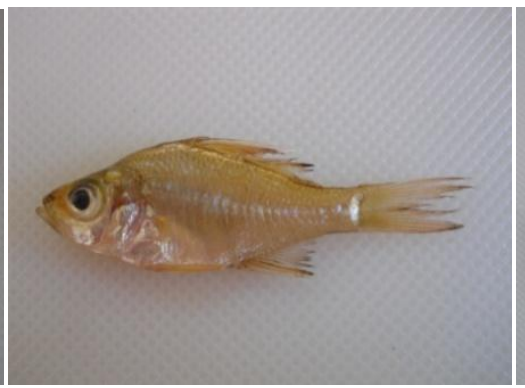

Fig.19. P. thomassi yellowish;

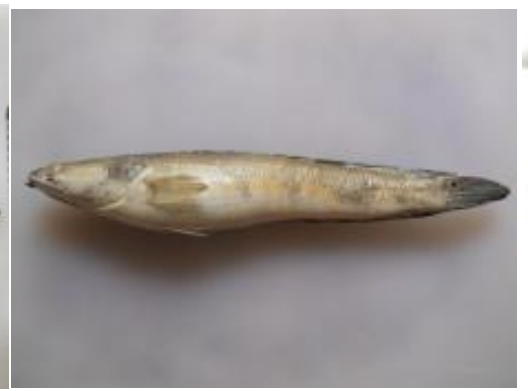

Fig. 22 C. marulius, whitish;

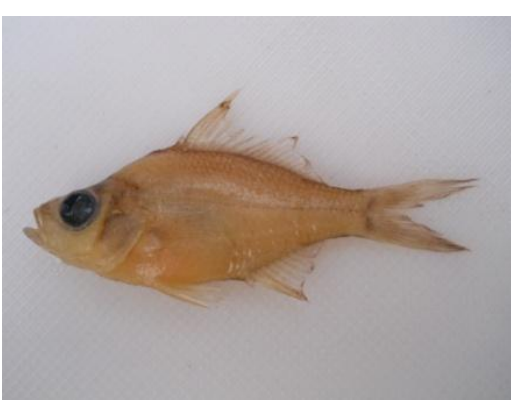

Fig.17 P. dayi, with deeper body

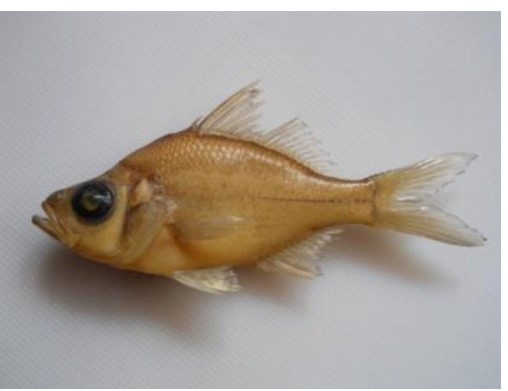

Fig.20. $P$. thomassi, preserved

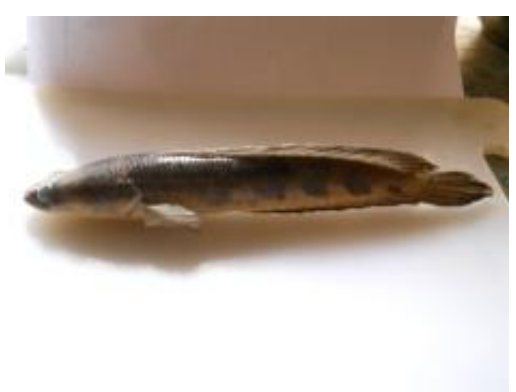

Fig.23. Preserved 


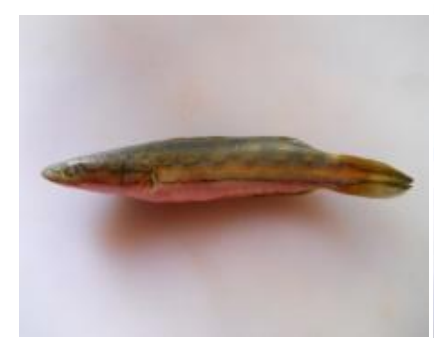

Fig.24. Channa micropeltes, young;

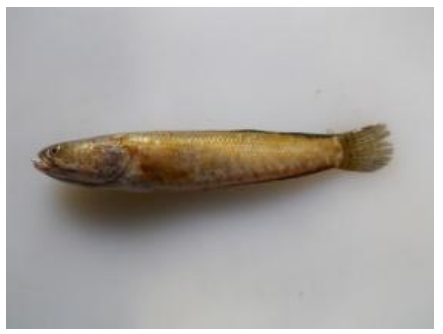

Fig. 2. Channa striatus, young

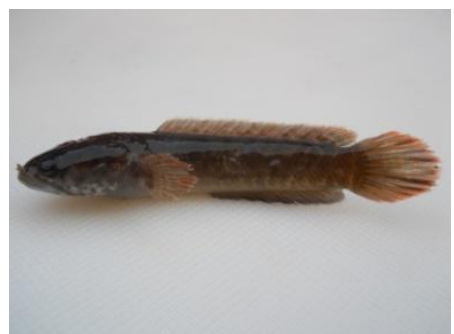

Fig.30. Channa gachua, fresh;

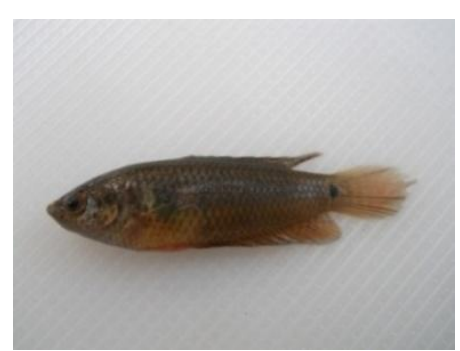

Fig.33. Pseudosphromenus cupanus, brownish; Fig. 34. Blackish;

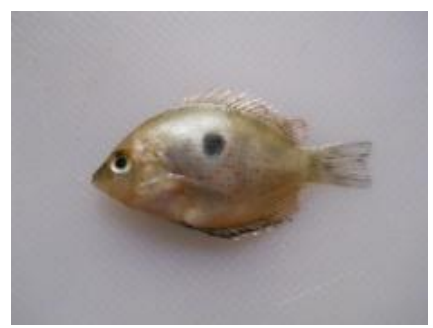

Fig.36. P. maculatus (Bloch), greenish

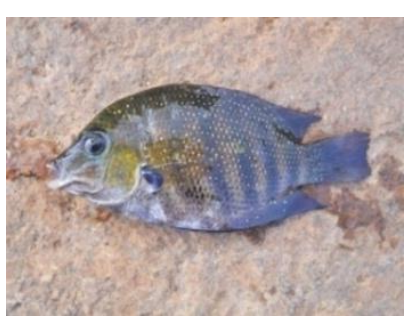

Fig.39. E. suratensis- dark green

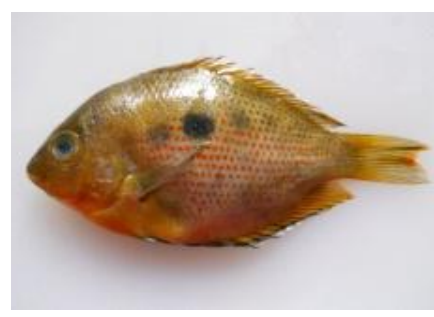

Fig.37 Golden

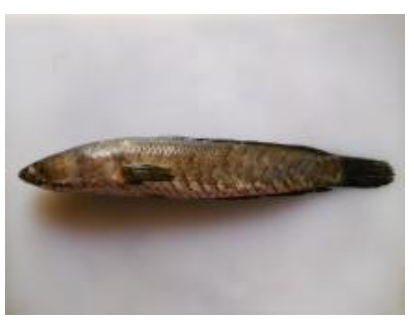

Fig. 28 C. striatus, adult

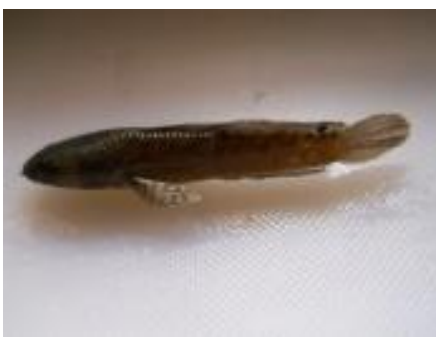

Fig. 31 C. gachua, preserved;

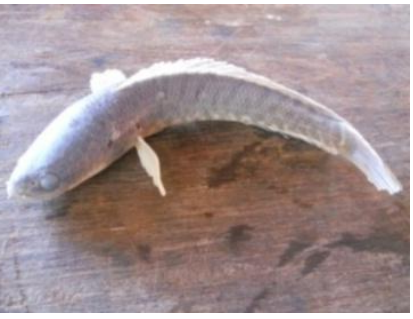

Fig. 29. C. striatus, preserved

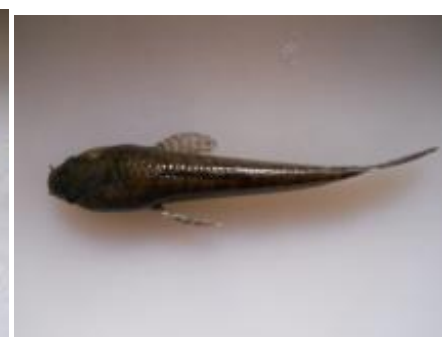

Fig.32. C. gachua, dorsal view

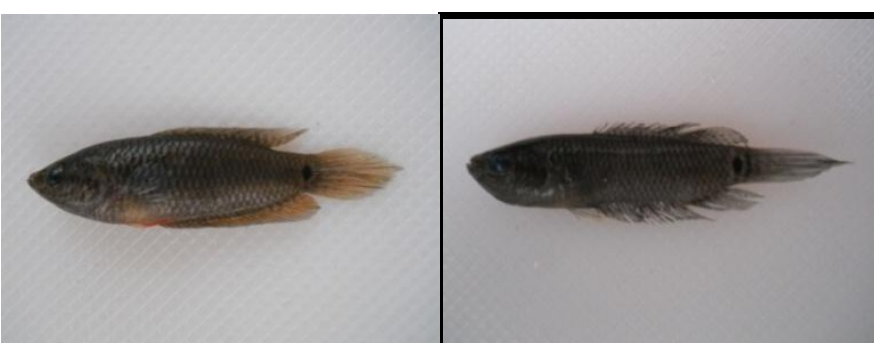

Fig. 35. Uncommon

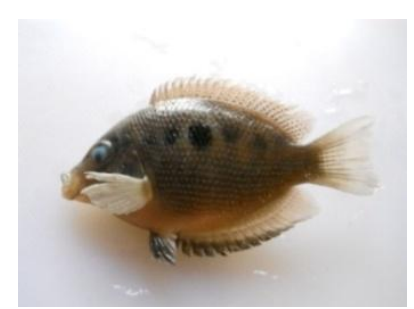

Fig.38. Preserved

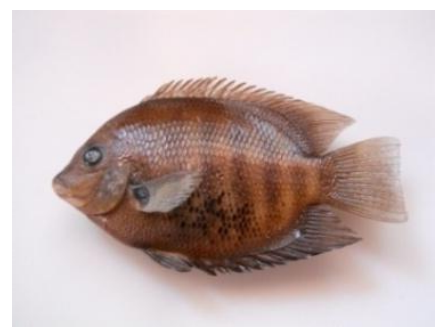

Fig.41. Preserved 


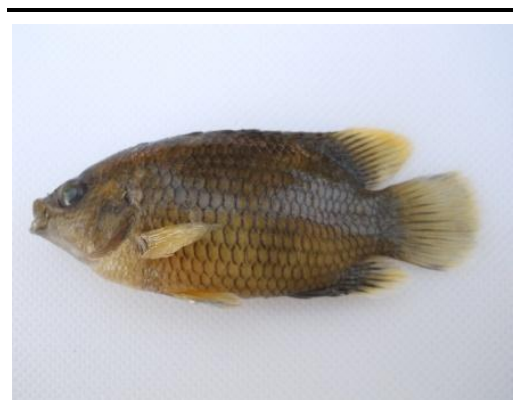

Fig.42. Pristolepis malabarica, fresh;

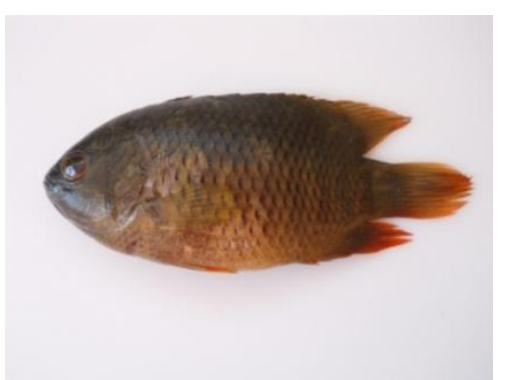

Fig.45 Pristolepis rubripinnis, reddish

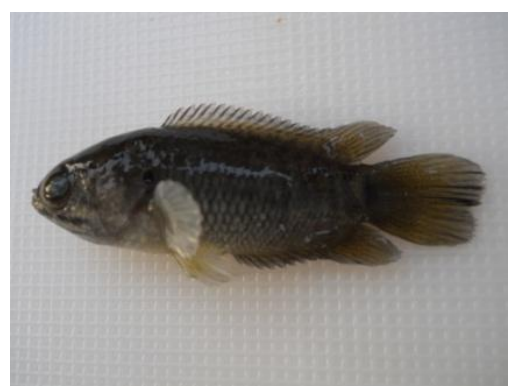

Fig. 48 Anabas testudineus, blackish

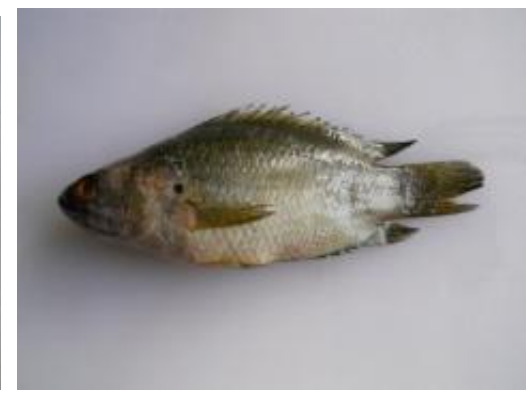

Fig.49 . Greenish;
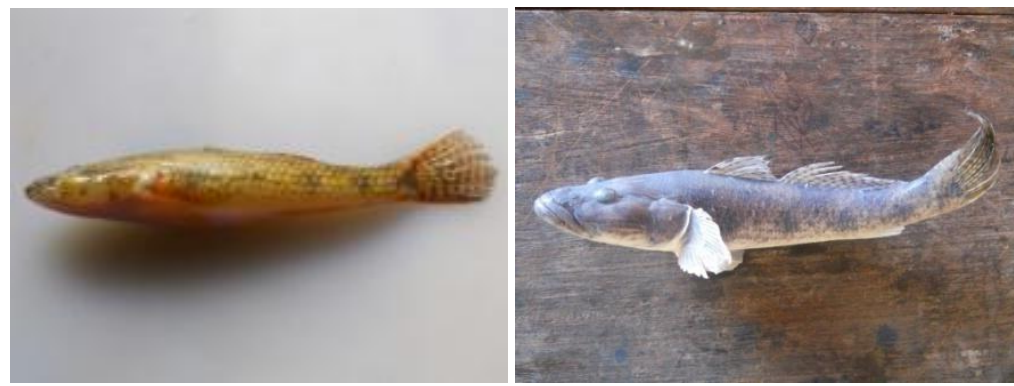

Fig.51. Glossogobius giuris giuris, yellowish

Fig.52. Preserved

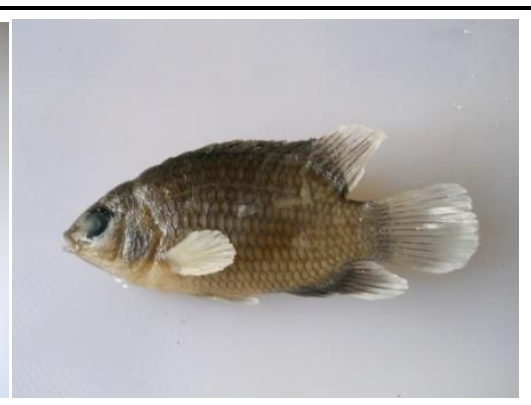

Fig.44. Greyish

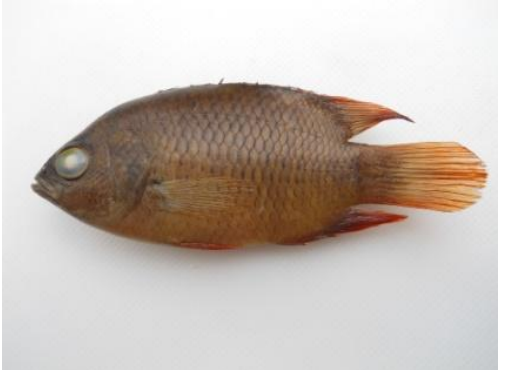

Fig.47. Preserved

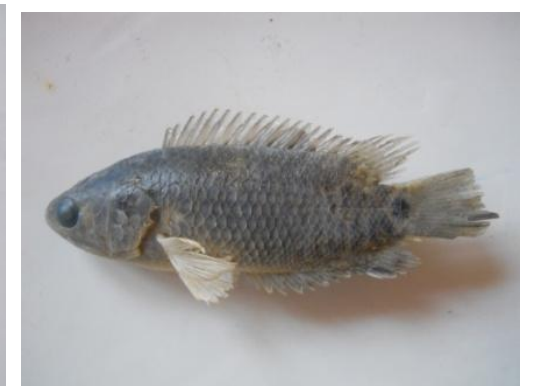

Fig.50. Preserved

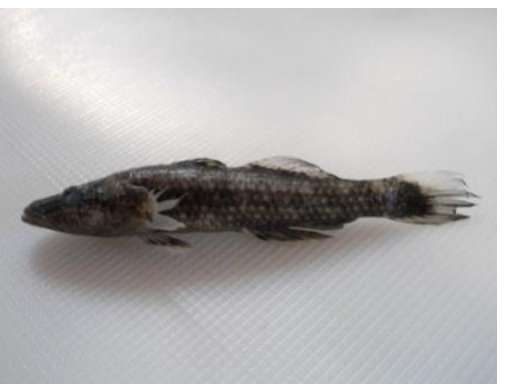

Fig.53. Blackish

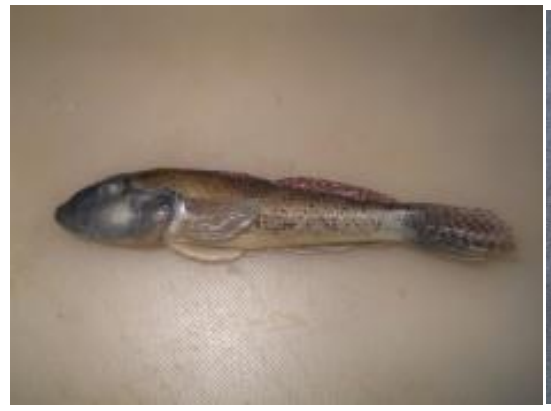

Fig.54. Awaous gutum- Brown

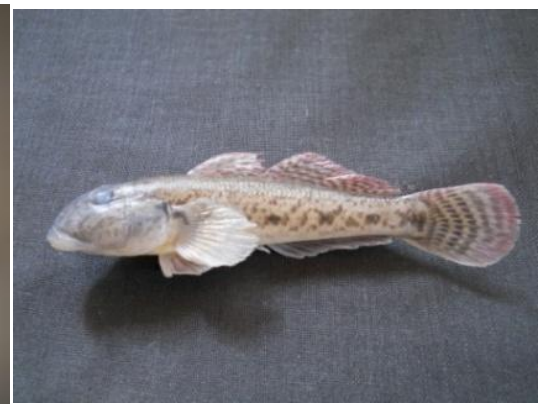

Fig.55. Reddish

\section{Conclusion}

Percoid fishes found in the inland water bodies of India are Snooks, Glass fishes, Leaf fishes, Cichlids, Gobioides, Climbing perches (Anabantids), Gouramies, Murrels (Talwar and A. Jhingran, 1991; Jayaram, 2010) etc. Lates, Ambassis, Chanda, Parambassis, Lutjanus, Nandus, Badis, 
Pristolepis, Terapon, Etroplus, Pseudetroplus, Oreochromis, Eleotris, Odonteleotris, Butis, Incara, Parachaeturichthys, Oxyurichthys,, Glossogobius, Bathygobius, Awaous, Brachygobius, Gobiopterus, Anabas, Ctenops, Pseudosphromenus, Colisa, Osphronemus, channa etc are the freshwater percoid fishes found in India (Talwar and Jhingran, 1991; Jayaram, 2010). Lates calcarifer, Ambassis commersoni, A. gymnocephalus, A. nalua, Chanda nama, Parambassis baculis, P. dayi, Parambassis lala, $P$. thomassi, P. ranga, Lutjanus johni, Nandus nandus, Pristolepis marginata, P. malabarica, $P$. rubripinnis, $P$. pentacantha, Etroplus suratensis, Pseudetroplus maculates, E. canarensis, Oreochromis mossambicus, O. nilotica, Glossogobius giuris, Awaous gutum, Anabas testudineus, Pseudosphromenus cupanus, $P$. dayi, Osphronemus goramy, Channa gachua, C. marulius, C. striatus, $C$. micropeltes, $C$. orientalis, $C$. punctatus etc are found in the freshwater bodies of Kerala.

Order Perciformes is the most diversified of all fish orders; its classification is controversial since many families are similar and are not easily definable in terms of common shared derived characters (Jayaram, 2010); taxonomic studies proved that meristic counts and morphometric characters of the examined fishes do not vary much from the standard values; morphological variations including colour changes found in many fishes etc. As there is no much meristic difference, no need of a detailed taxonomic analysis.

\section{ACKNOWLEDGEMENT}

The author acknowledges Principal, Baby John Memorial Government College, Chavara, Kollam for providing necessary facilities to undergo this study.

\section{REFERENCES}

[1] Easa PS, Shaji CP, 2003. Biodiversity documentation for Kerala, Kerala Forest Research Institute, Peechi.

[2] Jayaram KC, 2002. Fundamentals of fish taxonomy, Narendra publishing House, Delhi.

[3] Jayaram KC, 2010. Fresh water fishes of the Indian region, Narendra publishing House, Delhi.

[4] Lakshmi NR (2009) Impact of human interventions on inland fisheries, a case study from Manimala River, Kerala, India, MPhil dissertation submitted to the University of Kerala, Thiruvananthapuram.

[5] Nelson JS, 2006. Fishes of the World. Hoboken, NJ: John Wiley \& Sons. New York.

[6] Payne AI, 1986. The ecology of tropical lakes and rivers, John Wiley and sons, New York.

[7] Plamoottil M (2015a) Ichthyodiversity of Manimala River, Kerala, India. Journal of Zoological and Bioscience Research,, 2 (2): 26-34.

[8] Plamoottil M (2015b) Taxonomic Notes on exotic fishes of Manimala River, Kerala, India. Journal of Entomology and Zoology Studies, vol.3, no. 5, pp. 74-76.

[9] Plamoottil M (2016) Systematic notes on hill stream cyprinid fishes of Manimala River, Kerala, India. Journal of Zoological and Bioscience Research, 3 (1): 10- 14.

[10] Talwar PK, A Jhingran, 1991. Inland fishes of India and adjacent countries,. Oxford and IBH publishing Co. Pvt. Ltd. 